KCL-TH-95-04

q-alg/9506017

\title{
ON QUANTUM LIE ALGEBRAS AND QUANTUM ROOT SYSTEMS
}

\author{
Gustav W. Delius 周 and Andreas Hüffmann \\ Department of Mathematics, King's College London \\ Strand, London WC2R 2LS, Great Britain \\ e-mail: delius@mth.kcl.ac.uk and aha@mth.kcl.ac.uk \\ www: http://www.mth.kcl.ac.uk/delius
}

\begin{abstract}
As a natural generalization of ordinary Lie algebras we introduce the concept of quantum Lie algebras $\mathcal{L}_{q}(g)$. We define these in terms of certain adjoint submodules of quantized enveloping algebras $U_{q}(g)$ endowed with a quantum Lie bracket given by the quantum adjoint action. The structure constants of these algebras depend on the quantum deformation parameter $q$ and they go over into the usual Lie algebras when $q=1$.

The notions of q-conjugation and q-linearity are introduced. q-linear analogues of the classical antipode and Cartan involution are defined and a generalised Killing form, q-linear in the first entry and linear in the second, is obtained. These structures allow the derivation of symmetries between the structure constants of quantum Lie algebras.

The explicitly worked out examples of $g=s l_{3}$ and $s_{5}$ illustrate the results.
\end{abstract}

${ }^{*}$ On leave from Department of Physics, Bielefeld University, Germany 


\section{Introduction}

Lie algebras and their associated root systems play a pervasive role in the theory of classical integrable models. The great breakthrough in the quantization of these models has been the realization of the importance of the quantized enveloping algebras associated to these Lie algebras [16, 11, 12, 14. With the help of these quantized enveloping algebras it has been possible to derive many exact results for the full quantum theories.

In this paper we will deal not with the quantization of the enveloping algebras of Lie algebras but with the quantization of the Lie algebras themselves. Given the fact that most of the properties of classical integrable models are described by the structure of Lie algebras rather than their enveloping algebras, it is worthwhile to attempt to describe the quantum integrable models with quantum Lie algebras instead of quantized enveloping algebras. In section 2 we will describe the particular examples of quantum integrable theories which motivated this work.

A Lie algebra $g$ is naturally embedded into its universal enveloping algebra $U(g)$ as a submodule with respect to the adjoint action. The Lie bracket on $g$ is the restriction of the adjoint action of $U(g)$ to this submodule.

In the quantum case we are given the quantized enveloping algebra $U_{q}(g)$ and its quantum adjoint action on itself. We study those submodules of $U_{q}(g)$ which under the quantum adjoint action transform as the adjoint representation, following a remark in [20]. We endow these modules with the quantum Lie bracket induced by the quantum adjoint action. The resulting algebras are not all isomorphic. But among them there are always distinguished ones which share further important properties with their classical counterparts and it is these which we study in detail in this paper. The precise definition of these quantum Lie algebras is contained in Definition 3 .

There is a different approach to the quantization of Lie algebras present in the literature. It is based on the notion of bicovariant differential calculus on quantum groups [35, 3, 4, 18, 2, 27, 28, 30]. The resulting structures are braided Lie algebras as discussed by Majid [23]. Their shortcoming is that they do not have the same dimension as the corresponding classical Lie algebras except in the case of $g=g l_{n}$. For a discussion of this problem see [31]. For the case of $g=s l_{n}$ this problem has recently been solved by Sudbery and Lyubashenko [32].

This paper is structured as follows. In section 2 we briefly mention the features of affine Toda quantum field theories which motivated our search for a quantum deformation of Lie algebras and root systems. This section is included purely as a motivation. Section 3 contains some necessary preliminary material on Lie algebras and on quantum enveloping algebras. In order to introduce the concept of quantum Lie algebras we give in section 4 the very simple example of $\mathcal{L}_{h}\left(s l_{2}\right)$. In section 5 we give the beginnings of a general study of the structure of quantum Lie algebras. The standard tools provided by the general structure of quantum groups are com-

plemented with the notion of q-conjugation. It is this construction that allows us to exploit a generalisation of the classical Killing form, defined in section 5.3, to obtain the analogue of the Weyl canonical form of a Lie algebra in section 5.4. Relations and symmetries of the structure constants of the quantum Lie algebras in this basis 
are derived in section 5.5 and the quantum root space is investigated in section 5.6.

Finally the structure constants for the quantum Lie algebras associated with the Lie algebras $a_{2}\left(=s l_{2}\right)$ and $c_{2}(=s p(4)=s o(5))$ are given in section 6. The calculations were done on a computer using Mathematica [34. The results were obtained without using the general results of section 5 on the structure of quantum Lie algebras but are of course found to be in agreement with them. By the same methods we have also obtained the explicit results for the quantizations of the Lie algebras $a_{3}=s l_{4}$ and $g_{2}$. All the explicit calculations and results are available in the form of Mathematica notebooks at http://www.mth.kcl.ac.uk/delius/q-lie.htm] on the World Wide Web.

The straightforward determination of the explicit $q$-dependent structure constants of quantum Lie algebras $\mathcal{L}_{q}(g)$ is extremely tedious. We have therefore recently described a general method for obtaining them from the R-matrix of $U_{q}(g)$ [8]. This method had independently and earlier been derived in the formalism of differential calculus on quantum groups, see e.g. [27]. However in [8] it is applied to $g=g l_{n}$ and $g=s l_{n}$ for all $n$. The paper [9] establishes the existence and uniqueness of the quantum Lie algebras discussed here.

\section{Physical Motivation}

We want to start by giving the physical motivation which has led us to undertake the present study of quantum Lie algebras and quantum root systems. This section is meant purely as a motivation and is in no way needed in the rest of the paper.

This work has grown out of our desire to understand the exact results which have been obtained in quantum affine Toda theories. In these theories it has been possible to obtain the full quantum mass ratios and the exact S-matrices for the fundamental particles [5, 7]. Furthermore, Dorey [10] has found an elegant description of these results in terms of properties of the root systems of the underlying Lie algebras. While this description is exact for the cases where the affine root system is self-dual, the true quantum results in the case of non-self-dual root systems require certain deformations, with the deformation parameter depending on the product of Planck's constant and the coupling constant [7].

It is tempting to conjecture that the systematics of these deformations might be understandable in terms of the quantum root systems of quantum Lie algebras. However, a concept of quantum root systems associated to quantum Lie algebras has, to our knowledge, never been studied in the literature.

Affine Toda theories are massive integrable two dimensional relativistic field theories described by the Lagrangian density

$$
L[\phi]=b\left(\partial_{\mu} \phi, \partial^{\mu} \phi\right)+\frac{m^{2}}{\beta^{2}} b\left(e^{\beta \operatorname{ad} \phi} z_{1}, z_{-1}\right),
$$

where the bosonic field $\phi$ takes its values in the Cartan subalgebra of a simple Lie algebra $g, m^{2}$ is a mass scale, $\beta$ is the coupling constant, $b(.,$.$) is the Killing form$ on $g$. The $z_{ \pm 1}$ are cyclic elements of $g$ which in a standard notation can be expressed 


$$
z_{1}=\sum_{\alpha \in \bar{\Delta}} \sqrt{n_{\alpha}} x_{\alpha}, \quad z_{-1}=\sum_{\alpha \in \bar{\Delta}} \sqrt{n_{\alpha}} x_{-\alpha}
$$

where $\bar{\Delta}$ is the set $\Delta$ of simple roots extended by the root $\alpha_{0}$ which is minus the highest root (or the highest short root in the case of twisted Toda theories). The $n_{\alpha}$ are the Kac labels defined so that $n_{\alpha_{0}}=1$ and $\sum_{\alpha \in \bar{\Delta}} n_{\alpha} \alpha=0$. The classical masses of the fields can be read off the Lagrangian and their squares are found to be the eigenvalues of the matrix (written in terms of some basis $\left\{h_{i}\right\}$ of the Cartan subalgebra)

$$
M_{i j}^{2}=\sum_{\alpha \in \bar{\Delta}} n_{\alpha} \alpha\left(h_{i}\right) \alpha\left(h_{j}\right)
$$

Equivalent characterizations of the squares of the masses is as the eigenvalues of the adjoint action of $z_{1} z_{-1}$ on the Lie algebra or as the length squared of the projections of certain roots into the lowest eigenspace of the Coxeter element of the Weyl group. Numerically this typically leads to values (slight modifications depend on the particular Lie algebra $g$ )

$$
m_{a}^{2}=8 m^{2} \sin ^{2} \frac{a \pi}{2 h}, \quad h=\sum_{\alpha \in \bar{\Delta}} n_{\alpha} .
$$

where $a$ is the integer labeling the particle and $h$ is the (twisted) Coxeter number of $g$.

In the quantum theory these masses receive quantum corrections. However, when the dust settles, it turns out that the exact quantum masses are still given by the formula in (2.4) but with the Coxeter number $h$ replaced by a "quantum" Coxeter number $\mathrm{H}$. When the set $\bar{\Delta}$ is self-dual (i.e., if $\forall \alpha \in \bar{\Delta}$ also $2 \alpha / \alpha^{2} \in \bar{\Delta}$ ) this quantum Coxeter numer is equal to its classical value but in the non-selfdual case it is coupling constant dependent in the generic form

$$
H=h+c \frac{\beta^{2} \hbar / 2 \pi}{1+\beta^{2} \hbar / 4 \pi}
$$

where $c$ depends on the particular Lie algebra. Will it be possible to find a quantum Lie algebraic explanation for these mass formulae? In particular, is there a natural definition of a quantum Coxeter number?

The factorized S-matrices for the fundamental particles of affine Toda theories have been exactly determined by solving the equations arising from the bootstrap principle [5, 7]. Dorey [10] found that solutions to these very stringent bootstrap equations could be constructed by using the properties of the root systems of Lie algebras. These solutions describe the S-matrices of the self-dual Toda theories. They have the special property that the locations of the poles do not depend on $\hbar$. In addition to Dorey's solutions there is another set of solutions in which the pole locations depend on $\hbar$ through the quantum Coxeter number. These solutions give the S-matrices of non-self-dual Toda theories. Can the reason for the existence of these solutions be understood in terms of the properties of quantum root systems? 


\section{Preliminaries}

For background on Lie algebras see for example [26]. Let $g$ be a simple complex Lie algebra of rank $r, R$ the set of non-zero roots and $\alpha_{1}, \alpha_{2}, \ldots, \alpha_{r}$ its simple roots. Let $b: g \otimes g \rightarrow \mathbb{C}$ be the Killing form. Choose a basis $\hat{h}_{1}, \hat{h}_{2}, \ldots \hat{h}_{r}$ for the Cartan subalgebra $\mathcal{H}$ so that $b\left(\hat{h}_{i}, h\right)=\alpha_{i}(h) \forall h \in \mathcal{H}$. Choose root vectors $\hat{x}_{\alpha}$ so that $b\left(\hat{x}_{\alpha}, \hat{x}_{-\alpha}\right)=-1$. Then the Lie bracket relations take the Weyl canonical form

$$
\begin{aligned}
& {\left[\hat{h}_{i}, \hat{x}_{\alpha}\right]=-\left[\hat{x}_{\alpha}, \hat{h}_{i}\right]=\alpha\left(\hat{h}_{i}\right) \hat{x}_{\alpha}, \quad\left[\hat{h}_{i}, \hat{h}_{j}\right]=0} \\
& {\left[\hat{x}_{\alpha}, \hat{x}_{-\alpha}\right]=-\hat{h}_{\alpha}, \quad \text { where if } \alpha=\sum k_{i} \alpha_{i} \text { then } \hat{h}_{\alpha}=\sum k_{i} \hat{h}_{i},} \\
& {\left[\hat{x}_{\alpha}, \hat{x}_{\beta}\right]=N_{\alpha, \beta} \hat{x}_{\alpha+\beta} \quad \text { for } \beta \neq-\alpha \text { and } \alpha+\beta \in R .}
\end{aligned}
$$

The $N_{\alpha, \beta}$ are real numbers which can be determined entirely in terms of the root system. The scalar product on the root lattice is defined by

$$
\alpha \cdot \beta \equiv b\left(\hat{h}_{\alpha}, \hat{h}_{\beta}\right)=\alpha\left(\hat{h}_{\beta}\right) .
$$

The Weyl canonical basis is related to the Chevalley canonical basis by

$$
x_{ \pm \alpha}= \pm \sqrt{\frac{2}{\alpha \cdot \alpha}} \hat{x}_{ \pm \alpha}, \quad h_{i}=\frac{2}{\alpha \cdot \alpha} \hat{h}_{i}
$$

In the Chevalley basis all structure constants are integers. To generate the Lie algebra it is sufficient to consider the simple root vectors $x_{i}^{ \pm}=x_{ \pm \alpha_{i}}$. The relations are then

$$
\begin{aligned}
& {\left[h_{i}, h_{j}\right]=0, \quad\left[h_{i}, x_{j}^{ \pm}\right]= \pm a_{i j} x_{j}^{ \pm}, \quad\left[x_{i}^{+}, x_{j}^{-}\right]=\delta_{i j} h_{j},} \\
& \operatorname{ad}\left(x_{i}^{ \pm}\right)^{1-a_{i j}}\left(x_{j}^{ \pm}\right)=0 \quad \text { if } i \neq j .
\end{aligned}
$$

The last relations are the Serre relations. The adjoint action is defined by the Lie bracket $a d(x)(y)=[x, y]$ and $a_{i j}=2 \alpha_{i} \cdot \alpha_{j} / \alpha_{i} \cdot \alpha_{i}$ is the Cartan matrix.

The universal enveloping algebra $U(g)$ is the unital associative algebra over $\mathbb{C}$ with generators $x_{i}^{+}, x_{i}^{-}, h_{i}, 1 \leq i \leq r$ and relations (3.4) in which the Lie bracket is replaced by the commutator. The quantized eveloping algebra $U_{h}(g)$ is an algebra over $\mathbb{C}[[h]]$, the ring of formal power series in the indeterminate $h$, with the same set of generators but with the deformed relations

$$
\begin{aligned}
& {\left[h_{i}, h_{j}\right]=0, \quad\left[h_{i}, x_{j}^{ \pm}\right]= \pm a_{i j} x_{j}^{ \pm},} \\
& {\left[x_{i}^{+}, x_{j}^{-}\right]=\delta_{i j} \frac{q_{i}^{h_{i}}-q_{i}^{-h_{i}}}{q_{i}-q_{i}^{-1}}}
\end{aligned}
$$

and the quantum Serre relations

$$
\sum_{k=0}^{1-a_{i j}}(-1)^{k}\left[\begin{array}{c}
1-a_{i j} \\
k
\end{array}\right]_{q_{i}}\left(x_{i}^{ \pm}\right)^{k} x_{j}^{ \pm}\left(x_{i}^{ \pm}\right)^{1-a_{i j}-k}=0 \quad i \neq j .
$$

${ }^{\dagger}$ We have found [6] to be a generally reliable reference on quantum groups. Our $x_{i}^{ \pm}$are related to the $X_{i}^{ \pm}$of [6] by $x_{i}^{+}=k_{i}^{-1 / 2} X_{i}^{+}$and $x_{i}^{-}=X_{i}^{-} k_{i}^{1 / 2}$. 
Here $\left[\begin{array}{l}a \\ b\end{array}\right]_{q}$ are the q-binomial coefficients. We have defined $q_{i}=e^{d_{i} h}$ where $d_{i}$ are coprime integers such that $d_{i} a_{i j}$ is a symmetric matrix. We will use the notation $k_{i}=q_{i}^{h_{i}}$ and then the relations (3.5) take the form

$$
k_{i} k_{j}=k_{j} k_{i}, \quad k_{i} x_{j}^{ \pm} k_{i}^{-1}=q_{i}^{ \pm a_{i j}} x_{j}^{ \pm} \quad \text { and } \quad\left[x_{i}^{+}, x_{j}^{-}\right]=\delta_{i j} \frac{k_{i}-k_{i}^{-1}}{q_{i}-q_{i}^{-1}} .
$$

Note the technical point that in this paper we do not work with some rational form $U_{q}(g)$ but always with the algebra $U_{h}(g)$ defined over $\mathbb{C}[[h]]$. Indeed it can be seen from the example of $g=a_{2}$ that in general our quantum Lie algebras do not exist in the usual adjoint rational form but that one would have to use the simply-connected rational form.

The Hopf algebra structure of $U_{h}(g)$ is given by the comultiplication

$$
\begin{aligned}
\Delta\left(h_{i}\right) & =h_{i} \otimes 1+1 \otimes h_{i}, \\
\Delta\left(x_{i}^{ \pm}\right) & =x_{i}^{ \pm} \otimes q_{i}^{h_{i} / 2}+q_{i}^{-h_{i} / 2} \otimes x_{i}^{ \pm},
\end{aligned}
$$

the antipode

$$
S\left(h_{i}\right)=-h_{i}, \quad S\left(x_{i}^{ \pm}\right)=-q_{i}^{ \pm 1} x_{i}^{ \pm},
$$

and the counit

$$
\epsilon\left(h_{i}\right)=\epsilon\left(x_{i}^{ \pm}\right)=0 .
$$

The antipode does not square to the identity but rather

$$
S^{2}(a)=u a u^{-1} \quad \text { with } u=q^{2 h_{\rho}},
$$

where $q=e^{h}$ and $h_{\rho}$ is the element of the Cartan subalgebra determined by $b\left(h_{\rho}, h\right)=\rho(h) \forall h \in \mathcal{H}$ with $\rho$ being half the sum of the positive roots.

The Cartan involution $\theta$ is given by the same formulas as in the classical case

$$
\theta\left(x_{i}^{ \pm}\right)=x_{i}^{\mp}, \quad \theta\left(h_{i}\right)=-h_{i} .
$$

It is an algebra automorphism and a coalgebra antiautomorphism

$$
\Delta \cdot \theta=(\theta \otimes \theta) \cdot \Delta^{T}, \quad S \cdot \theta=\theta \cdot S^{-1} .
$$

If the Dynkin diagram of $g$ has a symmetry $\tau$ which maps node $i$ into node $\tau(i)$ then the Lie algebra $g$ has an automorphism

$$
\tau\left(x_{i}^{ \pm}\right)=x_{\tau(i)}^{ \pm}, \quad \tau\left(h_{i}\right)=h_{\tau(i)}
$$

which extends to a Hopf-algebra automorphism of $U_{h}(g)$. Such $\tau$ are refered to as diagram automorphisms and except for rescalings of the $x_{i}^{ \pm}$they are the only Hopf-algebra automorphisms of $U_{h}(g)$.

The adjoint action of $U_{h}(g)$ on itself, using Sweedler's notation [33], is given by

$$
x \circ y=\sum x_{(1)} y S\left(x_{(2)}\right), \quad x, y \in U_{h}(g) .
$$

There is a second adjoint action $\bullet$ defined by

$$
x \bullet y=\sum x_{(2)} y S^{-1}\left(x_{(1)}\right) .
$$

The Cartan involution $\theta$ and the antipode $S$ respect the adjoint actions in the sense of $[\theta(a) \bullet \theta(b)]=\theta([a \circ b])$ and $[S(a) \bullet S(b)]=S\left(\left[S^{-1}(a) \circ b\right]\right)$ for all $a, b \in U_{h}(g)$. 


\section{The $s l_{2}$ example}

As an introduction to the idea of a quantum Lie algebra it is useful to consider the very simple example of $s l_{2}$. The quantized enveloping algebra $U_{h}\left(s l_{2}\right)$ is generated by the three generators $h, x^{+}, x^{-}$and the commutation relations

$$
\left[h, x^{ \pm}\right]= \pm 2 x^{ \pm}, \quad\left[x^{+}, x^{-}\right]=\frac{q^{h}-q^{-h}}{q-q^{-1}} .
$$

Thus these three generators do not close to form a Lie algebra because the right hand side of the second equation is non-linear. Of course one would not expect them to do so. In the quantum case the commutator, which describes the classical adjoint action, should be replaced by the quantum adjoint action described in (3.16). In general the adjoint action on any $a \in U_{h}(g)$ is given by

$$
h \circ a=[h, a], \quad x^{ \pm} \circ a=x^{ \pm} a q^{-h / 2}-q^{-h / 2 \pm 1} a x^{ \pm} .
$$

and this produces the commutator only for $q=1$. The generators $h, x^{ \pm}$do not close even under the quantum adjoint action. However the elements

$$
X^{ \pm}=q^{h / 2} x^{ \pm}, \quad H=q^{-1} x^{+} x^{-}-q x^{-} x^{+}
$$

do. Indeed, their adjoint actions on each other can be easily calculated to be given by

$$
\begin{aligned}
{\left[H \circ X^{+}\right]=\left(1+q^{-2}\right) X^{+}, } & {\left[X^{+} \circ H\right]=-\left(1+q^{2}\right) X^{+}, } \\
{\left[H \circ X^{-}\right]=-\left(1+q^{2}\right) X^{-}, } & {\left[X^{-} \circ H\right]=\left(1+q^{-2}\right) X^{-}, } \\
{\left[X^{+} \circ X^{-}\right]=H, } & {\left[X^{-} \circ X^{+}\right]=-H, } \\
{[H \circ H]=\left(q^{-2}-q^{2}\right) H, } & {\left[X^{ \pm} \circ X^{ \pm}\right]=0 . }
\end{aligned}
$$

We use the bracket notation for the quantum adjoint action to indicate that we now view it as the quantum analoge of the Lie bracket. The algebra in (4.4) is the quantum Lie algebra $\mathcal{L}_{h}\left(s l_{2}\right)$. Its structure constants are $q$-dependent in such a way that it goes over into the classical $s l_{2}$ Lie algebra for $q=1$.

The simplicity of this example is deceptive. For any Lie algebra other than $s l_{2}$ the associated quantum Lie algebra is much more complex. We give other examples in section 6 .

\section{General structure}

It is now our aim to make some general statements about the structure of quantum Lie algebras and to derive symmetries between their structure constants.

\section{1 q-conjugation}

An important role is played in our general study by the concept of $q$-conjugation. 
Definition 1 a) $\underline{\text { q-conjugation }} \sim: \mathbb{C}[[h]] \rightarrow \mathbb{C}[[h]], a \mapsto \tilde{a}$ is the ring automorphism defined by $\tilde{h}=-h$.

b) Let $M, N$ be $\mathbb{C}[[h]]$-modules. A map $\phi: M \rightarrow N$ is q-linear if

$$
\phi(\lambda a)=\tilde{\lambda} \phi(a), \quad \forall a \in M, \lambda \in \mathbb{C}[[h]] .
$$

c) Let $A, B$ be algebras over $\mathbb{C}[[h]]$. A q-linear map $\phi: A \rightarrow B$ is an algebra q-homo$\underline{\text { morphism }}$ if it respects the algebra product, i.e., if $\forall a, a^{\prime} \in A, \phi\left(a \overline{\left.a^{\prime}\right)=\phi(a) \phi\left(a^{\prime}\right)}\right.$. q-anti-isomorphisms, q-automorphisms, etc., are defined analogously.

Note the analogy between the concepts of $q$-conjugation and complex conjugation and between $q$-linear maps and anti-linear maps.

Definition 2 q-conjugation on the quantum group $U_{h}(g)$ is the algebra q-automorphism $\sim: U_{h}(g) \rightarrow U_{h}(g)$ that extends q-conjugation on $\mathbb{C}[[h]]$ by acting as the identity on the generators $x_{i}^{ \pm}$and $h_{i}$.

This definition is consistent because the relations (3.5) and (3.6) are invariant under $q \mapsto q^{-1}$. The notion of q-conjugation has been introduced already in [13].

Defining a tilded Cartan involution and a tilded antipode as compositions

$$
\tilde{S}=\sim \cdot S, \quad \tilde{\theta}=\sim \cdot \theta,
$$

the concept of q-conjugation proves to be useful as we have

Lemma 1 a) q-conjugation is a Hopf algebra q-isomorphism $\sim: U_{h}(g) \rightarrow U_{h}(g)^{\mathrm{op}}$, in particular

$$
\epsilon \cdot \sim=\sim \cdot \epsilon, \quad \Delta \cdot \sim=\sim \cdot \Delta^{T}, \quad S \cdot \sim=\sim \cdot S^{-1} .
$$

b) q-conjugation relates the adjoint actions as

$$
\tilde{a} \bullet \tilde{b}=\widetilde{a \circ b}, \quad \forall a, b \in U_{h}(g) .
$$

c) $\tilde{\theta}: U_{h}(g) \rightarrow U_{h}(g)$ is a Hopf algebra q-isomorphism, especially

$$
\tilde{\theta}(a) \circ \tilde{\theta}(b)=\tilde{\theta}(a \circ b), \quad \forall a, b \in U_{h}(g) .
$$

d) $\tilde{S}: U_{h}(g) \rightarrow U_{h}(g)$ is an algebra q-anti-isomorphism such that

$$
\tilde{S}(a) \circ \tilde{S}(b)=\tilde{S}\left(S^{-1}(a) \circ b\right), \quad \forall a, b \in U_{h}(g) .
$$

\subsection{Quantum Lie algebras $\mathcal{L}_{h}(g)$}

A Lie algebra $g$ is naturally embedded into its universal enveloping algebra $U(g)$. It forms a subspace of the enveloping algebra which under the adjoint action transforms in the adjoint representation and the adjoint action restricts to the Lie bracket. As a starting point it is natural to define a quantum Lie algebra $\mathcal{L}_{h}(g)$ as a submodule of the quantized enveloping algebra $U_{h}(g)$ with the analogous property. The following definition additionally asks for a quantum Lie algebra to be invariant under $\tilde{\theta}, \tilde{S}$ and $\tau$ as this is not guaranteed by the classical limit itself.

While a modification of the following definition would be appropriate also to the case of Kac-Moody algebras, in this paper we have the case of finite dimensional Lie algebras in mind. 
Definition 3 A quantum Lie algebra $\mathcal{L}_{h}(g)$ associated to a finite-dimensional simple complex Lie algebra $g$ is a finite-dimensional indecomposable $\circ$ - submodule of $U_{h}(g)$ endowed with the quantum Lie bracket $[a \circ b]=a \circ b$ such that

1. $\mathcal{L}_{h}(g)$ is a deformation of $g$, i.e., $\left.\mathcal{L}_{h}(g)\right|_{h=0}=g$.

2. $\mathcal{L}_{h}(g)$ is invariant under $\tilde{\theta}, \tilde{S}$ and any diagram automorphism $\tau$.

An immediate consequence of this definition is that, under the adjoint action of $U_{h}(g), \mathcal{L}_{h}(g)$ transforms as the adjoint representation. The structure of this representation is well know. As is the case with all finite dimensional highest weight representations of $U_{h}(g)$ [24, 21], it is just a deformation of the corresponding classical representation. It follows in particular that $\mathcal{L}_{h}(g)$ splits into submodules of definite weight

$$
\mathcal{L}_{h}(g)=\bigoplus_{\alpha \in R} \mathcal{L}_{\alpha} \oplus \mathcal{L}_{0}, \quad h \circ a_{\alpha}=\alpha(h) a_{\alpha} \forall a_{\alpha} \in \mathcal{L}_{\alpha} .
$$

where the dimension of $\mathcal{L}_{0}$ is equal to the rank of $g$ and the $\mathcal{L}_{\alpha}$ are one-dimensional for any root $\alpha$ of $g$. (5.7) defines a grading of the quantum Lie algebra: $\left[\mathcal{L}_{\alpha} \circ \mathcal{L}_{\beta}\right] \in$ $\mathcal{L}_{\alpha+\beta}$. We will refer to $\mathcal{L}_{0}=\mathcal{H}$ as the Cartan subalgebra and to the elements of $\mathcal{L}_{\alpha}$ as root vectors.

We choose some basis $\left\{X_{\alpha} \mid \alpha \in R\right\} \cup\left\{H_{i} \mid i=1 \ldots \operatorname{rank}(\mathrm{g})\right\}$ for the quantum Lie algebra $\mathcal{L}_{h}(g)$ so that $X_{\alpha} \in \mathcal{L}_{\alpha}, H_{i} \in \mathcal{H}$. Because of the grading (5.7) the Lie bracket relations of $\mathcal{L}_{h}(g)$ are restricted to take the form

$$
\begin{aligned}
& {\left[H_{i} \circ X_{\alpha}\right]=l_{\alpha}\left(H_{i}\right) X_{\alpha}, \quad\left[X_{\alpha} \circ H_{i}\right]=-r_{\alpha}\left(H_{i}\right) X_{\alpha},} \\
& {\left[H_{i} \circ H_{j}\right]=f_{i j}{ }^{k} H_{k}, \quad\left[X_{\alpha} \circ X_{-\alpha}\right]=-H_{\alpha} \in \mathcal{L}_{0},} \\
& {\left[X_{\alpha} \circ X_{\beta}\right]=N_{\alpha \beta} X_{\alpha+\beta} \quad \text { for } \beta \neq-\alpha \text { and } \alpha+\beta \in R .}
\end{aligned}
$$

This is similar in form to the classical relations (3.1). There are however some crucial differences. Because the quantum Lie bracket is not anti-symmetric, there are two sets of roots, the 'left' roots $l_{\alpha}$ and the 'right' roots $r_{\alpha}$. Furthermore these roots are now not valued in $\mathbb{C}$ but in $\mathbb{C}[[h]]$. Similarly the constants $N_{\alpha, \beta}$ and $f_{i j}{ }^{k}$ are elements of $\mathbb{C}[[h]]$ \&. Note also that $\left[H_{i} \circ H_{j}\right]$ can be non-zero.

The requirement of invariance of $\mathcal{L}_{h}(g)$ under $\tilde{\theta}, \tilde{S}$ and $\tau$ is not empty. Already the example of $g=a_{2}$, treated in section [6.1, exhibits a whole family of $U_{h}(g) \circ$ submodules which satisfy the first part of definition 3 but not the second. However, given any non-invariant $U_{h}(g) \circ$ - submodule $\mathcal{L}_{h}(g)_{0} \subset U_{h}(g)$ satisfying the first part of the definition, a symmetrization with respect to $\tilde{\theta}, \tilde{S}$ and $\tau$ is always possible. To see this, fix a highest weight state $\psi \in \mathcal{L}_{h}(g)_{0}$ and choose a lowest weight state $\bar{\psi}=$ $P\left(x^{-}\right) \circ \psi, P\left(x^{-}\right)$being a monomial in the generators $x_{i}^{-} . \tilde{\theta}\left(\mathcal{L}_{h}(g)_{0}\right)$ satisfies the first part of the definition as well. Fix a highest weight state $\psi^{\prime} \in \tilde{\theta}\left(\mathcal{L}_{h}(g)_{0}\right)$ by requiring $\tilde{\theta}\left(\psi^{\prime}\right)=\bar{\psi}$ and define $\bar{\psi}^{\prime}=P\left(x^{-}\right) \circ \psi^{\prime}$. Then $\tilde{\theta}(\psi)=\alpha \bar{\psi}^{\prime}$ with some $0 \neq \alpha \in \mathbb{C}[[h]]$. The equalities $\psi=\tilde{\alpha} \tilde{P}\left(x^{+}\right) P\left(x^{-}\right) \circ \psi$ and $\psi^{\prime}=\alpha \tilde{P}\left(x^{+}\right) P\left(x^{-}\right) \circ \psi^{\prime}$ imply $\alpha=\tilde{\alpha}$. Due

\footnotetext{
$\ddagger$ It will usually be possible to treat $h$ as a numeric deformation parameter and then to work over $\mathbb{C}$. However before doing this it is clearly necessary to verify that all occuring power series in $h$ converge for a certain range of values for $h$.
} 
to the classical limit $\alpha$ has a square root. Renormalizing $Q\left(x^{-}\right)=\sqrt{\alpha} P\left(x^{-}\right), \phi=\psi$, $\bar{\phi}=Q\left(x^{-}\right) \circ \phi, \phi^{\prime}=\sqrt{\alpha} \psi^{\prime}$ and $\bar{\phi}^{\prime}=Q\left(x^{-}\right) \circ \phi^{\prime}$ leads to $\tilde{\theta}(\phi)=\bar{\phi}^{\prime}$ and $\tilde{\theta}\left(\phi^{\prime}\right)=\bar{\phi}$. $\phi_{1}=\gamma \phi+\tilde{\gamma} \phi^{\prime}$ satisfies $\tilde{\theta}\left(\phi_{1}\right)=Q\left(x^{-}\right) \circ \phi_{1}$ for $0 \neq \gamma \in \mathbb{C}[[h]]$ arbitrary but fixed.

Note that the above construction goes through under the additional requirement $\tilde{Q}\left(x^{-}\right)=q^{-4 \lambda\left(h_{\rho}\right)} Q\left(x^{-}\right), \lambda$ the highest root. This means $S^{2}\left(Q\left(x^{-}\right)\right)=\tilde{Q}\left(x^{-}\right)$. Now let $\phi_{2}=\phi_{1}-\tilde{S}\left(\phi_{1}\right)$ to find $\tilde{S}\left(\phi_{2}\right)=-\phi_{2}$ and, using (5.6), $\tilde{\theta}\left(\phi_{2}\right)=Q\left(x^{-}\right) \circ \phi_{2}$ as desired. Hence, $\mathcal{L}_{h}(g)=U_{h}(g) \circ \phi_{2}$ is a quantum Lie algebra. In case there is a diagram automorphism $\tau$ it is possible to additionally symmetrise with respect to $\tau$. $\tau$ then restricts to an automorphism of the resulting quantum Lie algebra.

Whenever there exists more than one quantum Lie algebras associated to the same Lie algebra $g$, then there exist also whole families of "almost" quantum Lie algebras which satisfy the first part of the definition but are not invariant under $\tilde{\theta}$. Consider the situation of two quantum Lie algebras with highest weight states $\psi_{1}, \psi_{2}$ such that $\tilde{\theta}\left(\psi_{j}\right)=Q\left(x^{-}\right) \circ \psi_{j}, j=1,2$. (An example for this situation is provided by $a_{2}$.) For $\alpha, \beta \in \mathbb{C}[[h]]$ construct the orbit $U_{h}(g) \circ\left(\alpha \psi_{1}+\beta \psi_{2}\right)$. From $\tilde{\theta}\left(\alpha \psi_{1}+\beta \psi_{2}\right)=Q\left(x^{-}\right) \circ\left(\tilde{\alpha} \psi_{1}+\tilde{\beta} \psi_{2}\right)$ it follows that $U_{h}(g) \circ\left(\alpha \psi_{1}+\beta \psi_{2}\right)$ is $\tilde{\theta}$-invariant only if $\frac{\alpha}{\beta}=\left(\frac{\alpha}{\beta}\right)$.

It had been observed already in the context of the bicovariant differential calculus that quantum Lie algebras are not left invariant by the antipode, see [29] for a discussion. We have just shown that it is however always possible to find quantum Lie algebras invariant under the combined action of the antipode and q-conjugation. This invariance will be crucial in the developments to follow.

There always exist a quantum Lie algebra $\mathcal{L}_{h}(g)$ associated to any simple complex Lie algebra $g$. Furthermore, all $\mathcal{L}_{h}(g)$ associated to the same $g$ are isomorphic as algebras. This has been shown in [9]

\subsection{Killing form}

The Killing form plays a crucial role in the structure theory of Lie algebras. It is a symmetric bilinear form on the Lie algebra and its crucial property is the invariance under the adjoint action. We define a quantum analogue.

Definition 4 The quantum Killing form is the map $B: \mathcal{L}_{h}(g) \otimes \mathcal{L}_{h}(g) \rightarrow \mathbb{C}[[h]]$ given by

$$
B(a, b)=-\operatorname{Tr}_{a d j}(\tilde{S}(a) b u) .
$$

Here $T r_{\text {adj }}$ denotes the trace over the adjoint representation and $u$ is the element of $U_{h}(g)$ expressing the square of the antipode as in (3.12).

This definition goes over into the classical Killing form $b$ in the classical limit $(h=0)$. From the non-degeneracy of the classical Killing form the non-degeneracy of the quantum Killing form follows. The analog of the ad-invariance for the quantum Killing form is

$$
B(a, c \circ b)=B(\tilde{S}(c) \circ a, b)
$$

which can be straightforwardly derived from the definition. 
Note that our quantum Killing form on $\mathcal{L}_{h}(g)$ is not the restriction of the usual Killing form $R$ on $U_{h}(g)$ first defined by Rosso [25]. The ad-invariance of the later is expressed in terms of the $U_{h}(g)$ coproduct: $\sum R\left(x_{(1)} \circ y, x_{(2)} \circ z\right)=\epsilon(x) R(y, z)$ $\forall x, y, z \in U_{h}(g)$. This is not useful for our purposes because the $U_{h}(g)$ coproduct leads out of the quantum Lie algebra $\mathcal{L}_{h}(g)$.

The quantum Killing form is $q$-linear in its first argument and linear in the second, i.e., for any $\lambda \in \mathbb{C}[[h]]$

$$
B(\lambda b, a)=\tilde{\lambda} B(b, a), \quad B(b, \lambda a)=\lambda B(b, a) .
$$

The quantum Killing form is not symmetric. However it is $q$-symmetric in the sense that

$$
B(b, a)=B \widetilde{(a, b)}
$$

In addition we have the following two relations

$$
\begin{aligned}
B(b, a) & =B(\tilde{S}(a), S(\tilde{b})) \\
& =B(\tilde{\theta}(a), \tilde{\theta}(b)) .
\end{aligned}
$$

To derive these relations one has to realize that the dual of $\pi_{a d j}, \pi_{a d j} \cdot \theta$ and $\sim \cdot \pi_{a d j} \cdot \sim$ are all related to the adjoint representation $\pi_{a d j}$ itself by similarity transformations.

\subsection{Weyl canonical form}

Proposition 2 It is possible to choose a basis $\left\{X_{\alpha} \mid \alpha \in R\right\} \cup\left\{H_{i} \mid i=1 \ldots \operatorname{rank}(g)\right\}$ for $\mathcal{L}_{h}(g)$ with the properties

$$
\begin{aligned}
& B\left(X_{\alpha}, X_{-\alpha}\right)=-1 \\
& \tilde{\theta}\left(X_{\alpha}\right)=X_{-\alpha}, \quad \tilde{\theta}\left(H_{i}\right)=-H_{i}, \\
& \tilde{S}\left(X_{\alpha}\right)=-q^{-\rho \cdot \alpha} X_{\alpha}, \quad \tilde{S}\left(H_{i}\right)=-H_{i} .
\end{aligned}
$$

Proof. In the following we will have to invert and to take square roots of elements of $\mathbb{C}[[h]]$. While this is not in general possible, it presents no problem for those formal power series which have a nonvanishing classical limit.

It is clear by (5.10) that $B\left(X_{\alpha}, X_{\beta}\right) \propto \delta_{\alpha+\beta, 0}$. We can choose the scale of the the $X_{\alpha}$ for positive $\alpha$ so that $B\left(X_{-\alpha}, X_{\alpha}\right)=-1$. By the symmetry property (5.12) of the Killing form the normalization (5.15) then holds for all $\alpha$.

The most general action of $\theta$ on $X_{\alpha}$ is, for reasons of weight, $\theta\left(X_{\alpha}\right)=f_{\alpha} \tilde{X}_{-\alpha}$ for some $f_{\alpha} \in \underset{\mathbb{C}}{\mathbb{C}}[[h]]$. Since $\theta^{2}=\mathrm{id}$ and $\sim$ commutes with $\theta$ we have $X_{\alpha}=f_{\alpha} \tilde{f}_{-\alpha} X_{\alpha}$, i.e. $f_{\alpha}^{-1}=\tilde{f}_{-\alpha}$ for all $\alpha$. If we rescale the $X_{\alpha}$ by $f_{\alpha}^{-1 / 2}$ both (5.16) and (5.15) hold. In particular, the $X_{\alpha}$ are determined up to sign by (5.15) and (5.16).

The most general action of $S$ on $X_{\alpha}$ is, again for reasons of weight, $S\left(X_{\alpha}\right)=$ $s_{\alpha} \tilde{X}_{\alpha}$ for some $s_{\alpha} \in \mathbb{C}[[h]]$. Hence $X_{\alpha}=S^{-1} \cdot S\left(X_{\alpha}\right)=s_{\alpha} S \widetilde{\left(X_{\alpha}\right)}=s_{\alpha} \tilde{s}_{\alpha} X_{\alpha}$, i.e. $s_{\alpha}^{-1}=\tilde{s}_{\alpha}$. Furthermore, $S \cdot \theta \cdot S=\theta$ and (5.16) imply $s_{-\alpha}=s_{\alpha}^{-1}$. Finally, (3.12), (5.11) and (5.13) lead to $1=-B\left(X_{-\alpha}, X_{\alpha}\right)=-B\left(\tilde{S}\left(X_{\alpha}\right), S\left(\tilde{X}_{-\alpha}\right)\right)=s_{\alpha} \tilde{s}_{-\alpha} q^{-2 \rho \cdot \alpha}$. Hence $s_{\alpha}^{2}=q^{2 \rho \cdot \alpha}$. The sign in (5.17) is determined by the classical limit $(h=0)$. 
We construct the basis states for the Cartan subalgebra $\mathcal{H}$ as follows

$$
H_{i}=\frac{1}{2}\left(q^{\rho \cdot \alpha_{i}}\left[X_{-\alpha_{i}} \circ X_{\alpha_{i}}\right]-q^{-\rho \cdot \alpha_{i}}\left[X_{\alpha_{i}} \circ X_{-\alpha_{i}}\right]\right) \text {. }
$$

Then, using (5.4) and (5.5), $\theta\left(H_{i}\right)=-\tilde{H}_{i}$ follows. The relation $S\left(H_{i}\right)=-\tilde{H}_{i}$ follows from $B\left(X_{-\alpha},\left[H_{i} \circ X_{\alpha}\right]\right)=B\left(\left[\tilde{\theta}\left(H_{i}\right) \circ \tilde{\theta}\left(X_{\alpha}\right)\right], \tilde{\theta}\left(X_{-\alpha}\right)\right)=-B\left(\left[H_{i} \circ X_{-\alpha}\right], X_{\alpha}\right)=$ $-B\left(X_{-\alpha},\left[\tilde{S}\left(H_{i}\right) \circ X_{\alpha}\right]\right)$. At $h=0$ the $H_{i}$ defined above are equal to the $\hat{h}_{i}$ of the usual Weyl canonical form of (3.1). This shows that the $H_{i}$ are linearly independent and thus give a basis of the Cartan subalgebra $\mathcal{H}$.

Remark. Note that the $H_{i}$ are not unique. For example, every choice $H_{i}=$ $\frac{1}{2}\left(\gamma_{i} H_{-\alpha_{i}}-\tilde{\gamma}_{i} H_{\alpha_{i}}\right)$ with $0 \neq \gamma_{i} \in \mathbb{C}[[h]]$ such that $\gamma_{i}+h \mathbb{C}[[h]]=1+h \mathbb{C}[[h]]$ is possible.

If $g$ has a diagram automorphism then $\tau$ acts as

$$
\tau\left(X_{\alpha}\right)=t_{\alpha} X_{\tau(\alpha)}, \quad \tau\left(H_{i}\right)=H_{\tau(i)}, \quad t_{\alpha}= \pm 1,
$$

where the signs $t_{\alpha}$ are the same as in the classical case. Proof. For reasons of weight $\tau\left(X_{\alpha}\right)=t_{\alpha} X_{\tau(\alpha)}$ for some $t_{\alpha} \in \mathbb{C}[[h]]$. From $B\left(X_{-\alpha}, X_{\alpha}\right)=B\left(\tau\left(X_{-\alpha}\right), \tau\left(X_{\alpha}\right)\right)=$

$\tilde{t}_{-\alpha} t_{\alpha} B\left(X_{-\alpha}, X_{\alpha}\right)$ it follows that $\tilde{t}_{-\alpha} t_{\alpha}=1$. From $\tilde{\theta}\left(\tau\left(X_{-\alpha}\right)\right)=\tau\left(\tilde{\theta}\left(X_{-\alpha}\right)\right)$ it follows that $\tilde{t}_{-\alpha}=t_{\alpha}$. Together this gives $t_{\alpha}^{2}=1$ and thus $t_{\alpha}= \pm 1$. The action on $H_{i}$ follows from (5.18) and the choice $t_{\alpha_{i}}=1$.

\subsection{Relations between structure constants}

We are now ready to derive relations between the various structure constants appearing in (5.8) when using the basis of proposition 2. From the isomorphism property (5.5) of $\theta$ we obtain an expression of the quantum roots for negative $\alpha$ in terms of the quantum roots for positive $\alpha$

$$
\begin{aligned}
& \left.\left[\tilde{\theta}\left(H_{i}\right) \circ \tilde{\theta}\left(X_{-\alpha}\right)\right]=\tilde{\theta}\left(\left[H_{i} \circ X_{-\alpha}\right]\right) \Rightarrow l_{-\alpha}\left(H_{i}\right)=-l_{\alpha} \widetilde{\left(H_{i}\right.}\right) \forall \alpha, i, \\
& {\left[\tilde{\theta}\left(X_{-\alpha}\right) \circ \tilde{\theta}\left(H_{i}\right)\right]=\tilde{\theta}\left(\left[X_{-\alpha} \circ H_{i}\right]\right) \Rightarrow r_{-\alpha}\left(H_{i}\right)=-r_{\alpha} \widetilde{\left(H_{i}\right)} \forall \alpha, i \text {. }}
\end{aligned}
$$

Thus, unlike in the classical case, the negative of a left quantum root is not a left quantum root again, but the q-conjugated negative is. Idem for right quantum roots. We also obtain relations for the structure constants $N$ and $f$

$$
\begin{aligned}
& {\left[\tilde{\theta}\left(X_{\alpha}\right) \circ \tilde{\theta}\left(X_{\beta}\right)\right]=\tilde{\theta}\left(\left[X_{\alpha} \circ X_{\beta}\right]\right) \Rightarrow N_{\alpha, \beta}=\tilde{N}_{-\alpha,-\beta} \quad \forall \alpha, \beta,} \\
& {\left[\tilde{\theta}\left(H_{i}\right) \circ \tilde{\theta}\left(H_{j}\right)\right]=\tilde{\theta}\left(\left[H_{i} \circ H_{j}\right]\right) \Rightarrow f_{i j}{ }^{k}=-\tilde{f}_{i j}{ }^{k} \quad \forall i, j, k .}
\end{aligned}
$$

From the ad-invariance (5.10) of the quantum Killing form we obtain the characterization of the Cartan subalgebra elements $H_{\alpha}$ in terms of the right roots

$$
\begin{aligned}
-B\left(H_{\alpha}, H\right) & =B\left(\left[X_{\alpha} \circ X_{-\alpha}\right], H\right)=B\left(X_{-\alpha},\left[\tilde{S}\left(X_{\alpha}\right) \circ H\right]\right) \\
& =B\left(X_{-\alpha},-q^{-\rho \cdot \alpha}\left[X_{\alpha} \circ H\right]\right)=q^{-\rho \cdot \alpha} r_{\alpha}(H) \quad \forall \alpha, \forall H \in \mathcal{H} .
\end{aligned}
$$

Because of the non-degeneracy of the Killing form these relations determine the $H_{\alpha}$ uniquely in terms of the roots. We also obtain further relations for the structure 
constants $N$ and $f$

$$
\begin{gathered}
B\left(\left[\tilde{S}\left(X_{\alpha}\right) \circ X_{\beta}\right], X_{-\alpha-\beta}\right)=B\left(X_{\beta},\left[X_{\alpha} \circ X_{-\alpha-\beta}\right]\right) \\
\Rightarrow N_{\alpha,-\alpha-\beta}=-q^{\rho \cdot \alpha} \tilde{N}_{\alpha, \beta} \quad \forall \alpha, \beta, \\
B\left(\left[\tilde{S}\left(H_{j}\right) \circ H_{i}\right], H_{k}\right)=B\left(H_{i},\left[H_{j} \circ H_{k}\right]\right) \\
\Rightarrow \sum_{l} f_{j k}{ }^{l} B_{i l}=-\sum_{l} \tilde{f}_{j i}^{l} B_{l k},
\end{gathered}
$$

where we have defined $B_{i j}=B\left(H_{i}, H_{j}\right)$.

There exists a quantum Lie algebra anti-automorphism $\chi: \mathcal{L}_{h}(G) \rightarrow \mathcal{L}_{h}(g)$ acting on the basis as

$$
\chi\left(X_{\alpha}\right)=-X_{-\alpha}, \quad \chi\left(H_{i}\right)=H_{i} .
$$

From the anti-automorphism property

$$
[\chi(a) \circ \chi(b)]=\chi([b \circ a]) \quad \forall a, b \in \mathcal{L}_{h}(g),
$$

we obtain the relation between the 'left' and 'right' quantum roots

$$
l_{\alpha}=-r_{-\alpha} \quad \forall \alpha,
$$

and the relations

$$
N_{\alpha, \beta}=-N_{-\beta,-\alpha}, \quad f_{i j}{ }^{k}=f_{j i}{ }^{k} .
$$

The proof that (5.27) defines an anti-automorphism of the quantum Lie algebras is contained in [9].

If $g$ has a diagram automorphism $\tau$ then this leads to further relations

$$
\begin{array}{lc}
f_{\tau(i) \tau(j)}{ }^{\tau(k)}=f_{i j}{ }^{k}, & N_{\tau(\alpha), \tau(\beta)}=t_{\alpha} t_{\beta} t_{\alpha+\beta} N_{\alpha, \beta}, \\
l_{\tau(\alpha)}\left(H_{\tau(i)}\right)=l_{\alpha}\left(H_{i}\right), & r_{\tau(\alpha)}\left(H_{\tau(i)}\right)=r_{\alpha}\left(H_{i}\right), \\
B_{\tau(i) \tau(j)}=B_{i j} . &
\end{array}
$$

\subsection{Quantum root spaces}

We have seen that a quantum Lie algebra posesses two sets of quantum roots, $l_{\alpha}$ and $r_{\alpha}$, defined by

$$
\left[H \circ X_{\alpha}\right]=l_{\alpha}(H) X_{\alpha}, \quad\left[X_{\alpha} \circ H\right]=-r_{\alpha}(H) X_{\alpha} .
$$

The roots are linear forms on the Cartan subalgebra $\mathcal{H}$ with values in $\mathbb{C}[[h]]$, i.e., they are elements of $\mathcal{H}^{*}$. If the quantum Lie algebra has the anti-automorphism $\chi$ of (5.27), then the roots are related by $r_{\alpha}=-l_{-\alpha}$, i.e., the set of right roots is just the negative of the set of left roots.

From the Killing form on $\mathcal{H}$ we construct a form on $\mathcal{H}^{*}$ in the usual way. To any element $v \in \mathcal{H}^{*}$ we associate the unique element $H_{v} \in \mathcal{H}$ satisfying $v(H)=$ $B\left(H_{v}, H\right) \forall H \in \mathcal{H}$. Note that this pairing is $q$-linear in the sense that the element 
of $\mathrm{H}$ associated to $\lambda v$ for some $\lambda \in \mathbb{C}[[h]]$ is not $\lambda H_{v}$ but $\tilde{\lambda} H_{v}$. The form on $\mathcal{H}^{*}$ is defined by

$$
\langle v, w\rangle=B\left(H_{v}, H_{w}\right) \quad \forall v, w \in \mathcal{H}^{*}
$$

Because the Killing form is $q$-linear in the first factor and linear in the second, the form $\langle.,$.$\rangle is linear in the first factor and q$-linear in the second

$$
\langle\lambda v, w\rangle=\lambda\langle v, w\rangle, \quad\langle v, \lambda w\rangle=\tilde{\lambda}\langle v, w\rangle .
$$

It is also $q$-symmetric

$$
\langle v, w\rangle=\langle\widetilde{w, v}\rangle
$$

From the relation (5.24) we can read off that, for example,

$$
\left\langle r_{\alpha}, r_{\beta}\right\rangle=q^{\rho \cdot(\alpha-\beta)} B\left(H_{\alpha}, H_{\beta}\right)
$$

In the classical case of complex Lie algebras one introduces a real form $\mathcal{H}_{\mathbb{R}}$ of the Cartan subalgebra and on its dual space $\mathcal{H}_{\mathbb{R}}^{*}$, which is a real vectorspace, the form induced by the Killing form is a real, positive definite, bilinear form, thus giving $\mathcal{H}_{\mathbb{R}}^{*}$ the structure of a Euclidean space. This is the root space.

We can imitate this construction for quantum Lie algebras. We define the 'qreal' form $\mathcal{H}_{\mathbb{R}\left[\left[h^{2}\right]\right]}$ of the Cartan subalgebra as the module over $\mathbb{R}\left[\left[h^{2}\right]\right]$ spanned by the $H_{i}$. We choose $\mathbb{R}\left[\left[h^{2}\right]\right]$ as the base ring because it consists of the elements of $\mathbb{C}[[h]]$ which are invariant under both complex conjugation and $q$-conjugation. The roots, when restricted to $\mathcal{H}_{\mathbb{R}}\left[\left[h^{2}\right]\right]$ still give values in $\mathbb{R}[[h]]$, and not in $\mathbb{R}\left[\left[h^{2}\right]\right]$, and thus do not lie in $\left(\mathcal{H}_{\mathbb{R}_{\left[\left[h^{2}\right]\right]}}\right)^{*}$. The $q$-symmetrized combinations $a_{\alpha}=\frac{1}{2}\left(r_{\alpha}-r_{-\alpha}\right)$ do however give values in $\mathbb{R}\left[\left[h^{2}\right]\right]$. The $a_{i} \equiv a_{\alpha_{i}}$ for all simple roots $\alpha_{i}$ form a basis for $\mathcal{H}_{\mathbb{R}\left[\left[h^{2}\right]\right]}^{*}$. On this basis the form is given by

$$
\left\langle a_{i}, a_{j}\right\rangle=B\left(H_{i}, H_{j}\right)=B_{i j} .
$$

We see immediately that the form $\langle.,$.$\left.\rangle restricted to \mathcal{H}_{\mathbb{R}}^{*}\left[h^{2}\right]\right]$ is a symmetric, nondegenerate, bilinear form with values in $\mathbb{R}\left[\left[h^{2}\right]\right]$.

We expect however that in an eventual axiomatic description of quantum root systems the unrestricted form $\langle.,$.$\rangle will be used and that the fact that it is not$ symmetric and bilinear but rather q-symmetric and q-bilinear will play a central role.

\section{$6 \quad$ Explicit examples}

We have explicitly constructed three examples of quantum Lie algebras, namely those associated to $g=a_{2}, a_{3}, c_{2}$ and to $g_{2}$. The construction follows straigthforwardly from the definition 3. We search for a highest weight state inside $U_{h}^{\geq 0}(g)$ and impose a symmetry constraint if appropriate. Then the corresponding orbit is constructed and explicitly tested for the invariance properties required by the definition to be satisfied. The details for the cases of $g=a_{2}$ and $c_{2}$ are given below. 
Rather than to describe the quantum groups in terms of fundamental generators and their relations, the selection of a Poincare Birkoff Witt (PBW) type basis is useful for explicit computations. In the construction of such a basis with the help of the Lusztig automorphisms [22] we follow the conventions of [6]; an alternative would be the approach of 19. For a reduced decomposition of the longest Weyl group element $w_{0}=s_{i_{1}} \ldots s_{i_{N}}$ the quantum root vectors are given by

$$
\begin{aligned}
e_{k} & =T_{i_{1}} \ldots T_{i_{(k-1)}}\left(X_{i_{k}}^{+}\right) \quad \text { and } \\
f_{k} & =T_{i_{1}} \ldots T_{i_{(k-1)}}\left(X_{i_{k}}^{-}\right) .
\end{aligned}
$$

Note that $e_{k}$ is a polynomial in $\left\{X_{i}^{+}\right\}$while analogously $f_{k}$ is a polynomial in $\left\{X_{i}^{-}\right\}$, although this is not entirely obvious from the definition of the Lusztig automorphisms $T_{j}$.

\section{$6.1 \quad \mathcal{L}_{h}\left(a_{2}\right)$}

$a_{2}=s l_{3}$ is the rank 2 Lie algebra with Cartan matrix

$$
a=\left(\begin{array}{cc}
2 & -1 \\
-1 & 2
\end{array}\right)
$$

It has a diagram automorphism $\tau$ which exchanges the two simple roots, i.e., $X_{1}^{ \pm} \leftrightarrow$ $X_{2}^{ \pm}$and $k_{1} \leftrightarrow k_{2}$. The quantum root vectors generating the PBW basis which we use involve the choice $w_{0}=s_{1} s_{2} s_{1}$ :

$$
\begin{array}{ll}
e_{1}=X_{1}^{+}, & e_{2}=-X_{1}^{+} X_{2}^{+}+q^{-1} X_{2}^{+} X_{1}^{+}, \quad e_{3}=X_{2}^{+}, \\
f_{1}=X_{1}^{-}, & f_{2}=q X_{1}^{-} X_{2}^{-}-X_{2}^{-} X_{1}^{-},
\end{array}
$$

The diagram automorphism acts as $\tau\left(e_{1}\right)=e_{3}, \tau\left(e_{2}\right)=-q^{-1} e_{2}-\left(1-q^{-2}\right) e_{3} e_{1}$, $\tau\left(e_{3}\right)=e_{1}$. In terms of the PBW basis it is straightforward to write down an Ansatz $\Psi$ for a highest weight state according to point 2) of the definition 3. Once we restrict the Ansatz for $\Psi$ to lie entirely in $U_{h}^{\geq 0}$, i.e.,not to contain any $f_{i}$, we find two independent solutions of the equations $x_{i}^{+} \circ \Psi=0$. With respect to the diagram automorphism these can be described as a highest weight state

$$
\Psi^{+}=e_{2}\left(k_{1}^{1 / 3} k_{2}^{-1 / 3}-q^{-1} k_{1}^{-1 / 3} k_{2}^{1 / 3}\right)-\left(1-q^{-2}\right) e_{3} e_{1} k_{1}^{-1 / 3} k_{2}^{1 / 3}
$$

that is invariant under the diagram symmetry, while

$$
\Psi^{-}=e_{2}\left(k_{1}^{1 / 3} k_{2}^{-1 / 3}+q^{-1} k_{1}^{-1 / 3} k_{2}^{1 / 3}\right)+\left(1-q^{-2}\right) e_{3} e_{1} k_{1}^{-1 / 3} k_{2}^{1 / 3}
$$

changes sign under the diagram automorphism. The (skew)invariance of $\Psi^{ \pm}$follows by means of $\left[e_{1}, e_{3}\right]_{q^{-1}}=-e_{2}$. The symmetrisation with respect to $\tau$ enforces the symmetries required by the definition of a quantum Lie algebra.

We now observe that $\Psi^{+}$vanishes in the classical limit $q \rightarrow 1$ whilst $\Psi^{-}$reduces to the highest root vector of the classical Lie algebra. Hence $\Psi^{-}$is a desirable starting point for the construction of an adjoint orbit. The resulting orbit is in fact found to satisfy all the requirements of definition 3. We then chose a quantum 
Weyl basis with the properties of proposition 2. The explicit expressions for these quantum Lie algebra generators are listed below to give the reader an idea about the form of these generators. Note for example that the quantum Cartan subalgebra generators are not simple expressions.

$$
\begin{gathered}
X_{\alpha_{1}+\alpha_{2}}=-C\left(e_{2}\left(q^{-1 / 2} k_{1}^{1 / 3} k_{2}^{-1 / 3}+q^{-3 / 2} k_{1}^{-1 / 3} k_{2}^{1 / 3}\right)-\left(q^{-1}-q\right) q^{-3 / 2} e_{3} e_{1} k_{1}^{-1 / 3} k_{2}^{1 / 3}\right) \\
X_{\alpha_{2}}=-i C\left(e_{3}\left(q^{1 / 2} k_{1}^{-2 / 3} k_{2}^{-1 / 3}+q^{-1 / 2} k_{1}^{2 / 3} k_{2}^{1 / 3}\right)+\left(q^{-1}-q\right) q^{-1 / 2} e_{2} f_{1} k_{1}^{1 / 3} k_{2}^{-1 / 3}\right) \\
X_{\alpha_{1}}=i C\left(e_{1}\left(q^{1 / 2} k_{1}^{-1 / 3} k_{2}^{-2 / 3}+q^{-1 / 2} k_{1}^{1 / 3} k_{2}^{2 / 3}\right)-\left(q^{-1}-q\right) q^{-3 / 2} e_{2} f_{3} k_{1}^{-1 / 3} k_{2}^{1 / 3}\right. \\
\left.+\left(q^{-1}-q\right)^{2} q^{-3 / 2} e_{3} e_{1} f_{3} k_{1}^{-1 / 3} k_{2}^{1 / 3}\right) \\
H_{1}=C^{2} \frac{1+q^{3}}{2(1-q)}\left(-q k_{1}^{2 / 3} k_{2}^{-2 / 3}+k_{1}^{-2 / 3} k_{2}^{+2 / 3}-k_{1}^{4 / 3} k_{2}^{2 / 3}+q k_{1}^{-4 / 3} k_{2}^{-2 / 3}\right. \\
+\left(1-q^{2}\right)^{2}\left(+e_{1} f_{1}\left(q^{-2} k_{1}^{-1 / 3} k_{2}^{-2 / 3}+q^{-3} k_{1}^{1 / 3} k_{2}^{2 / 3}\right)\right. \\
\left.+q^{-4} e_{2} f_{2} k_{1}^{-1 / 3} k_{2}^{1 / 3}-q^{-2} e_{3} f_{3} k_{1}^{2 / 3} k_{2}^{1 / 3}\right) \\
\left.-\left(1-q^{2}\right)^{3} q^{-5} e_{3} e_{1} f_{2} k_{1}^{-1 / 3} k_{2}^{1 / 3}\right) \\
H_{2}=C^{2} \frac{1+q^{3}}{2(1-q)}\left(-q k_{1}^{-2 / 3} k_{2}^{2 / 3}+k_{1}^{2 / 3} k_{2}^{-2 / 3}-k_{1}^{2 / 3} k_{2}^{4 / 3}+q k_{1}^{-2 / 3} k_{2}^{-4 / 3}\right. \\
+\left(1-q^{2}\right)^{2}\left(+e_{3} f_{3}\left(q^{-2} k_{1}^{-2 / 3} k_{2}^{-1 / 3}+q^{-3} k_{1}^{2 / 3} k_{2}^{1 / 3}\right)\right. \\
\left.+q^{-4} e_{2} f_{2} k_{1}^{1 / 3} k_{2}^{-1 / 3}-q^{-2} e_{1} f_{1} k_{1}^{1 / 3} k_{2}^{2 / 3}\right) \\
\left.+\left(1-q^{2}\right)^{3} q^{-4} e_{2} f_{3} f_{1} k_{1}^{1 / 3} k_{2}^{-1 / 3}\right) \\
X_{-\alpha_{1}}=i C\left(f_{1}\left(q^{1 / 2} k_{1}^{2 / 3} k_{2}^{-2 / 3}+q^{-1 / 2} k_{1}^{4 / 3} k_{2}^{2 / 3}\right)-\left(q^{-1}-q\right) q^{-1 / 2} e_{3} f_{2} k_{1}^{2 / 3} k_{2}^{1 / 3}\right) \\
X_{-\alpha_{2}}=-i C\left(f_{3}\left(q^{1 / 2} k_{1}^{-2 / 3} k_{2}^{2 / 3}+q^{-1 / 2} k_{1}^{2 / 3} k_{2}^{4 / 3}\right)+\left(q^{-1}-q\right) q^{-3 / 2} e_{1} f_{2} k_{1}^{1 / 3} k_{2}^{2 / 3}\right. \\
\left.+\left(q^{-1}-q\right)^{2} q^{-1 / 2} e_{1} f_{3} f_{1} k_{1}^{1 / 3} k_{2}^{2 / 3}\right)
\end{gathered}
$$

The normalisation factor is

$$
C=\left(2\left(q^{-1 / 2}+q^{1 / 2}\right)\left(q^{-3 / 2}+q^{3 / 2}\right)\left(q^{-3}+q^{-1}-1+q+q^{3}\right)\right)^{-1 / 2} .
$$

It could be absorbed into a different normalization of the quantum Killing form in (5.9).

The left quantum roots are, using the notation $H=\sum h_{i} H_{i}$,

$$
\begin{aligned}
l_{\alpha_{1}}(H) & =l\left(\left(q^{-3 / 2}+q^{-1 / 2}\right) h_{1}-q^{1 / 2} h_{2}\right) \\
l_{\alpha_{2}}(H) & =l\left(-q^{1 / 2} h_{1}+\left(q^{-3 / 2}+q^{-1 / 2}\right) h_{2}\right) \\
l_{\alpha_{1}+\alpha_{2}}(H) & =l q^{-3 / 2}\left(h_{1}+h_{2}\right) \\
& \quad l=C^{2}\left(q^{-1 / 2}+q^{1 / 2}\right)\left(q^{-3 / 2}+q^{3 / 2}\right)^{2} / 2
\end{aligned}
$$

The negative roots are obtained by $q$-conjugation according to (5.20). The right roots are given according to (5.29). The roots are seen to be related by the diagram automorphism according to 5.32 
The q-conjugation-invariant roots $a_{\alpha}=\frac{1}{2}\left(r_{\alpha}+l_{\alpha}\right)$ introduced in section 5.6 are

$$
\begin{aligned}
a_{\alpha_{1}} & =\frac{l}{2}\left(\left(q^{-3 / 2}+q^{-1 / 2}+q^{1 / 2}+q^{3 / 2}\right) h_{1}-\left(q^{-1 / 2}+q^{1 / 2}\right) h_{2}\right) \\
a_{\alpha_{2}} & =\frac{l}{2}\left(-\left(q^{-1 / 2}+q^{1 / 2}\right) h_{1}+\left(q^{-3 / 2}+q^{-1 / 2}+q^{1 / 2}+q^{3 / 2}\right) h_{2}\right) \\
a_{\alpha_{1}+\alpha_{2}} & =\frac{l}{2}\left(q^{-3 / 2}+q^{3 / 2}\right)\left(h_{1}+h_{2}\right)
\end{aligned}
$$

These have the classical properties

$$
a_{\alpha}+a_{\beta}=a_{\alpha+\beta}, \quad a_{-\alpha}=-a_{\alpha},
$$

i.e., they form a root lattice. This interesting feature, which makes these root systems look very similar to their classical counterparts, is true for $g=a_{n}$ for any $n$ [8], but is not true for $c_{2}$, as we will see in the next section.

The Killing form on the Cartan subalgebra is given by the matrix $B$ with entries $B_{i j}=B\left(H_{i}, H_{j}\right)=\left\langle a_{\alpha_{i}}, a_{\alpha_{j}}\right\rangle$,

$$
\begin{aligned}
B & =b\left(\begin{array}{cc}
q+q^{-1} & -1 \\
-1 & q+q^{-1}
\end{array}\right) \\
b & =\left(\left(q^{-1 / 2}+q^{1 / 2}\right)^{2}\left(q^{-3 / 2}+q^{3 / 2}\right)^{2}\right) C^{2} / 4
\end{aligned}
$$

The pairwise equality of the elements is due to the diagram automorphism.

Once one has the knowledge of the Killing form and of the roots, the $H_{\alpha}$, which appear as the result of $\left[X_{\alpha} \circ X_{-\alpha}\right]$, are determined by (5.24). In terms of the $H_{i}$ they read

$$
\begin{aligned}
& H_{\alpha_{1}}=a\left(-q^{-1 / 2} H_{1}+\left(-q^{1 / 2}+q^{3 / 2}\right) H_{2}\right) \\
& H_{\alpha_{2}}=a\left(\left(-q^{1 / 2}+q^{3 / 2}\right) H_{1}-q^{-1 / 2} H_{2}\right) \\
& H_{\alpha_{1}+\alpha_{2}}=-a q^{1 / 2}\left(H_{1}+H_{2}\right) \\
& H_{-\alpha_{1}}=a\left(q^{1 / 2} H_{1}+\left(q^{-1 / 2}-q^{-3 / 2}\right) H_{2}\right) \\
& H_{-\alpha_{2}}=a\left(\left(q^{-1 / 2}-q^{-3 / 2}\right) H_{1}+q^{1 / 2} H_{2}\right) \\
& H_{-\alpha_{1}-\alpha_{2}}=a q^{-1 / 2}\left(H_{1}+H_{2}\right) \\
& a=2\left(q^{-3 / 2}+q^{3 / 2}\right)^{-1}
\end{aligned}
$$

Note that the coefficients in the expansion of the $H_{\alpha}$ are related to those in $H_{-\alpha}$ by q-conjugation and sign change.

We need to give only one of the structure constants $N$

$$
N_{\alpha_{1}, \alpha_{2}}=\left(q^{-3 / 2}+q^{3 / 2}\right) C
$$

Through the relations (5.25) and (5.30) all the other non-zero $N_{\alpha, \beta}$ are related to this (note that $N_{\alpha_{1}, \alpha_{2}}=\tilde{N}_{\alpha_{1}, \alpha_{2}}$ )

$$
\begin{aligned}
& N_{\alpha_{1},-\alpha_{1}-\alpha_{2}}=-q N_{\alpha_{1}, \alpha_{2}}, \quad N_{\alpha_{2}, \alpha_{1}}=-N_{\alpha_{1}, \alpha_{2}}, \quad N_{\alpha_{2},-\alpha_{1}-\alpha_{2}}=q N_{\alpha_{1}, \alpha_{2}} \\
& N_{\alpha_{1}+\alpha_{2},-\alpha_{1}}=q N_{\alpha_{1}, \alpha_{2}}, \quad N_{\alpha_{1}+\alpha_{2},-\alpha_{2}}=-q N_{\alpha_{1}, \alpha_{2}}, \quad N_{-\alpha_{1},-\alpha_{2}}=N_{\alpha_{1}, \alpha_{2}} \\
& N_{-\alpha_{1}, \alpha_{1}+\alpha_{2}}=-q^{-1} N_{\alpha_{1}, \alpha_{2}}, \quad N_{-\alpha_{2}, \alpha_{1}+\alpha_{2}}=q^{-1} N_{\alpha_{1}, \alpha_{2}}, \quad N_{-\alpha_{2},-\alpha_{1}}=-N_{\alpha_{1}, \alpha_{2}} \\
& N_{-\alpha_{1}-\alpha_{2}, \alpha_{1}}=q^{-1} N_{\alpha_{1}, \alpha_{2}}, \quad N_{-\alpha_{1}-\alpha_{2}, \alpha_{2}}=-q^{-1} N_{\alpha_{1}, \alpha_{2}}
\end{aligned}
$$


This is confirmed by the results of the explicit calculations.

For the structure constants $f_{i j}{ }^{k}$ for the Cartan subalgebra we find

$$
\begin{aligned}
f_{11}{ }^{1}= & f_{22}{ }^{2}=-f\left(q^{-2}+q^{-1}+1+q+q^{2}\right) \\
f_{22}{ }^{1}= & f_{11}{ }^{2}=-f\left(q^{-1}+q\right) \\
f_{12}{ }^{1}= & f_{21}{ }^{1}=f_{12}{ }^{2}=f_{21}{ }^{2}=f \\
& \quad f=\left(q^{1 / 2}-q^{-1 / 2}\right)\left(q^{-1 / 2}+q^{1 / 2}\right)^{2}\left(q^{-3 / 2}+q^{3 / 2}\right) C^{2} / 2
\end{aligned}
$$

\section{$6.2 \mathcal{L}_{h}\left(c_{2}\right)$}

$c_{2}=s p(4)=b_{2}=s o(5)$ is the rank 2 Lie algebra with Cartan matrix

$$
a=\left(\begin{array}{cc}
2 & -2 \\
-1 & 2
\end{array}\right)
$$

It has no diagram automorphisms. With conventions analogous to the previous example

$$
\begin{aligned}
l_{\alpha_{1}}(H)= & l\left(\left(q^{-2}-1+q^{2}\right)^{2} q^{-1} h_{1}-q^{3} h_{2}\right) \\
l_{\alpha_{2}}(H)= & l\left(-\left(q^{-2}-1+q^{2}\right) q^{-1} h_{1}+\left(q^{-1}+q\right) q^{-2} h_{2}\right) \\
l_{\alpha_{1}+\alpha_{2}}(H)= & l\left(\left(q^{-1}-q\right)\left(q^{-2}-1+q^{2}\right) q^{-2} h_{1}+q^{-1} h_{2}\right) \\
l_{2 \alpha_{1}+\alpha_{2}}(H)= & l\left(\left(q^{-2}-1+q^{2}\right) q^{-3} h_{1}\right) \\
& l=\left(q^{-1}+q\right)^{3}\left(q^{-2}-1+q^{2}\right)^{2} C^{2} / 2 \\
a_{\alpha_{1}}(H)= & a\left(\left(q^{-2}-1+q^{2}\right)^{2} h_{1}-\left(q^{-2}-1+q^{2}\right) h_{2}\right) \\
a_{\alpha_{2}}(H)= & a\left(-\left(q^{-2}-1+q^{2}\right) h_{1}+\left(q^{-2}+q^{2}\right) h_{2}\right) \\
a_{\alpha_{1}+\alpha_{2}}(H)= & a\left(\left(q^{-1}-q\right)^{2}\left(q^{-2}-1+q^{2}\right) h_{1}+h_{2}\right) \\
a_{2 \alpha_{1}+\alpha_{2}}(H)= & a\left(\left(q^{-2}-1+q^{2}\right)^{2} h_{1}\right) \\
& a=\left(q^{-1}+q\right) C^{2} / 2
\end{aligned}
$$

The normalisation constant is

$$
\begin{array}{r}
C=\left(\left(q^{-1}+q\right)^{2}\left(q^{-2}+q^{2}\right)\left(q^{-1}+1+q\right)\left(q^{-1}-1+q\right)\right. \\
\left.\left(q^{-2}-1+q^{2}\right)\left(q^{-4}-q^{-2}+1-q^{2}+q^{4}\right)\right)^{-1 / 2} .
\end{array}
$$

Note that

$$
a_{2 \alpha_{1}+\alpha_{2}} \neq a_{\alpha_{1}+\alpha_{2}}+a_{\alpha_{1}}
$$

Thus, in contrast to the case of $g=a_{n}$, these roots do not form a root lattice.

\footnotetext{
$\S$ It is tempting to speculate that there may be a relation between the non-closure of the above root triangle and the non-closure of some mass triangles in the affine Toda theory based on $c_{2}$.
} 
The Killing form on the Cartan subalgebra is given by the matrix $B$ with entries $B_{i j}=B\left(H_{i}, H_{j}\right)=\left\langle a_{\alpha_{i}}, a_{\alpha_{j}}\right\rangle$,

$$
\begin{aligned}
B & =b\left(\begin{array}{cc}
q^{-2}-1+q^{2} & -1 \\
-1 & \frac{q^{-2}+q^{2}}{q^{-2}-1+q^{2}}
\end{array}\right) \\
b & =\left(\left(q^{-1}+q^{1}\right)^{4}\left(q^{-2}-1+q^{2}\right)^{3}\right) C^{2} / 4
\end{aligned}
$$

We find

$$
N_{\alpha, \beta}=-\left(q^{-1}+q\right)\left(q^{-2}-1+q^{2}\right) n_{\alpha, \beta}
$$

with the $n_{\alpha, \beta}$ given in the following table. The rows are labeled by $\alpha$ and the coloums by $\beta$.

\begin{tabular}{r|cccccccc} 
& $\begin{array}{c}2 \alpha_{1} \\
+\alpha_{2}\end{array}$ & $\begin{array}{c}\alpha_{1} \\
\alpha_{2}\end{array}$ & $\alpha_{2}$ & $\alpha_{1}$ & $-\alpha_{1}$ & $-\alpha_{2}$ & $-\alpha_{1}$ & $-2 \alpha_{1}$ \\
\hline $2 \alpha_{1}+\alpha_{2}$ & 0 & 0 & 0 & 0 & $-q^{2}$ & 0 & $q^{2}$ & $-\alpha_{2}$ \\
$\alpha_{1}+\alpha_{2}$ & 0 & 0 & 0 & $q$ & $q^{3}$ & -1 & 0 & $-q^{2}$ \\
$\alpha_{2}$ & 0 & 0 & 0 & $-q^{2}$ & 0 & 0 & 1 & 0 \\
$\alpha_{1}$ & 0 & $-q^{-1}$ & $q^{-2}$ & 0 & 0 & 0 & $-q^{3}$ & $q^{2}$ \\
$-\alpha_{1}$ & $q^{-2}$ & $-q^{-3}$ & 0 & 0 & 0 & $q^{2}$ & $-q$ & 0 \\
$-\alpha_{2}$ & 0 & 1 & 0 & 0 & $-q^{-2}$ & 0 & 0 & 0 \\
$-\alpha_{1}-\alpha_{2}$ & $-q^{-2}$ & 0 & -1 & $q^{-3}$ & $q^{-1}$ & 0 & 0 & 0 \\
$-2 \alpha_{1}-\alpha_{2}$ & 0 & $q^{-2}$ & 0 & $-q^{-2}$ & 0 & 0 & 0 & 0
\end{tabular}

In view of the relations given in section 5.5 the structure constants are fixed once $N_{\alpha_{1}, \alpha_{2}}$ and $N_{\alpha_{1}, \alpha_{1}+\alpha_{2}}$ are given. Also because of these relations the above table is qanti-symmetric about the diagonal and antisymmetric about the opposite diagonal.

For the structure constants $f_{i j}^{k}$ for the Cartan subalgebra we find

$$
\begin{aligned}
f_{11}{ }^{1}= & -f\left(q^{-2}-1+q^{2}\right)\left(q^{-4}-q^{-2}+3-q^{2}+q^{4}\right) \\
f_{22}{ }^{2}= & -f\left(q^{-2}-q^{-1}+1-q+q^{2}\right)\left(q^{-2}+q^{-1}+1+q+q^{2}\right) \\
f_{11}{ }^{2}= & -f\left(q^{-2}+q^{2}\right)\left(q^{-2}-1+q^{2}\right)^{2} \\
f_{22}{ }^{1}= & -f\left(q^{-2}+q^{2}\right)\left(q^{-2}-1+q^{2}\right)^{-1} \\
f_{12}{ }^{2}= & f_{21}{ }^{2}=f\left(q^{-2}+q^{2}\right)\left(q^{-2}-1+q^{2}\right) \\
f_{12}{ }^{1}= & f_{21}{ }^{1}=f \\
& \quad f=-\left(q^{-1}-q\right)\left(q^{-1}+q\right)^{3}\left(q^{-2}-1+q\right) / 2
\end{aligned}
$$

The the quantum roots corresponding to the positive classical roots are represented by

$$
\begin{gathered}
H_{\alpha_{1}}=d\left(\left(-q^{-4}+q^{-2}-2+q^{2}\right) H_{1}-\right. \\
\left.\left(q^{-1}-q\right)\left(q^{-2}+q^{2}\right)\left(q^{-2}-1+q^{2}\right) q^{-1} H_{2}\right) \\
H_{\alpha_{2}}=d\left(-\left(q^{-1}-q\right) q^{2} H_{1}-\left(q^{-2}-1+q^{2}\right) q^{-1} H_{2}\right) \\
H_{\alpha_{1}+\alpha_{2}}=d\left(\left(-q^{-2}+1-2 q^{2}-q^{4}\right) H_{1}-\left(q^{-2}-1+q^{2}\right) H_{2}\right) \\
H_{2 \alpha_{1}+\alpha_{2}}=d\left(-\left(q^{-2}+q^{2}\right) q H_{1}-\left(q^{-2}-1+q^{2}\right) q H_{2}\right) \\
d=2\left(\left(q^{-1}+q\right)\left(q^{-2}-1+q^{2}\right)\right)^{-1}
\end{gathered}
$$


Again the corresponding expressions for negative $\alpha$ are obtained by q-conjugating the coefficients and changing the sign.

\section{Discussion}

We have shown that it is possible to develop a theory of quantum Lie algebras in terms of an analogue of Weyl's canonical form and the resulting quantum roots and structure constants. The key idea is the concept of q-conjugation that allows us to exploit q-linear analogues of the antipode and the Cartan involution in connection with a generalised Killing form.

Objects similar to our quantum Lie algebras have been studied in the framework of bicovariant differential calculus on quantum groups, see [2] for a very readable review. There one considers the dual space to the space of left-invariant one-forms, which is a o-submodule of $U_{h}(g)$. The case $g=s l_{3}$ has been explicitly worked out in [1]. It does not coincide with our quantum Lie algebra $\mathcal{L}_{h}\left(s l_{3}\right)$ studied in section 6.1. In particular the module of [1] is not invariant under the diagram automorphism of $s l_{3}$.

The q-conjugation $\sim$ acting on $U_{h}(g)$ which we have defined in definition 2 does not restrict to $\mathcal{L}_{h}(g)$. We can however define a different q-conjugation on $\mathcal{L}_{h}(g)$.

Definition 5 q-conjugation on $\mathcal{L}_{h}(g)$ is the q-linear map $\mathcal{L}_{h}(g) \rightarrow \mathcal{L}_{h}(g)$, a $\mapsto a^{q}$ which extends the q-conjugation $\sim$ on $\mathbb{C}[[h]]$ by acting as the identity on the basis elements $X_{\alpha}$ and $H_{i}$.

The quantum Lie bracket $[a \circ b]$ which we have defined through the adjoint action in $U_{h}(g)$ is clearly not anti-symmetric, i.e. $[a \circ b] \neq-[b \circ a]$. However we have

Theorem 3 The quantum Lie bracket is q-anti-symmetric in the sense that

$$
\left[a^{q} \circ b^{q}\right]=-[b \circ a]^{q}, \quad \forall a, b \in \mathcal{L}_{h}(g), \quad g=a_{n}, c_{2} .
$$

This follows from combining the antiautomorphism $\chi$, described in equation (5.27), with the $q$-isomorphism $\tilde{\theta}: a^{q}=-\chi(\tilde{\theta}(a))$.

Our observations in this paper regarding the structure of quantum Lie algebras have raised many new questions. Among them: • What is the origin of the q-antisymmetry (7.1) of the quantum Lie bracket? This has recently been answered in [9]. - What are representations of quantum Lie algebras? - How can the q-symmetric q-bilinear form $\langle.,$.$\rangle on root space defined in (5.34) be used to define a q-geometry$ on root space? What are q-Weyl "reflections" with respect to such a form? Can they be used to define quantum root systems axiomatically? - Is there a connection to quantum affine Toda theory and other quantum integrable models? These questions are under investigation.

Finally we would like to draw the readers attention to the work of Sudbery and Lyubashenko [32 which has appeared since the completion of this work. They also

\footnotetext{
『Rather than working with modules over $\mathbb{C}[[h]]$ people treat $q=\exp (h)$ as a number and work with vector spaces over $\mathbb{C}$ or $\mathbb{R}$.
} 
give quantum Lie algebras for $s l_{2}$ and $s l_{3}$. For $s l_{3}$ however they do not impose invariance under the diagram automorphism.

For further information on quantum Lie algebras visit the quantum Lie algebra home page on the World Wide Web at http://www.mth.kcl.ac.uk/delius/q-lie.html.

Acknowledgements G.W.D. thanks the Deutsche Forschungsgemeinschaft for a Habilitationsstipendium. A.H. thanks the EC for a research fellowship. We have profited greatly from discussions with Mark Gould and Yao-Zhong Zhang which have led to the work in [8]. G.W.D. thanks Ed Corrigan and Patrick Dorey for discussions and hospitality during his visit in Durham.

\section{References}

[1] P. Aschieri, L. Castellani, Bicovariant differential geometry of the quantum group $G L_{q}(3)$, Phys. Lett. B293 (1992) 299.

[2] P. Aschieri, L. Castellani, An introduction to noncommutative differential geometry on quantum groups, Int. J. Mod. Phys. A8 (1993) 1667, hep-th/9207084.

[3] D. Bernard, Quantum Lie Algebras and Differential Calculus on Quantum Groups, Prog. Theo. Phys. Suppl. 102 (1990) 49.

[4] D. Bernard, A remark on quasi-triangular quantum Lie algebras, Phys. Lett. B260 (1991) 389.

[5] H.W. Braden, E. Corrigan, P.E. Dorey, R. Sasaki, Affine Toda field theory and exact S-matrices, Nucl. Phys. B338 (1990) 689.

[6] V. Chari, A. Pressley, A Guide to Quantum Groups, Cambridge University Press (1994).

[7] G.W. Delius, M.T. Grisaru, D. Zanon, Exact S-matrices for nonsimply-laced affine Toda theories, Nucl. Phys. B382 (1992) 365, hep-th/9201067.

[8] G.W. Delius, A. Hüffmann, M.D. Gould Y.-Z. Zhang, Quantum Lie algebras associated to $U_{q}\left(g l_{n}\right)$ and $U_{q}\left(s l_{n}\right)$, q-alg/9508013.

[9] G.W. Delius, M.D. Gould, Quantum Lie algebras, their existence, uniqueness and q-antisymmetry, KCL-TH-96-05.

[10] P.E. Dorey, Root systems and purely elastic S-matrices, Nucl. Phys. B358 (1991) 654.

[11] V.G. Drinfel'd, Hopf algebras and the quantum Yang-Baxter equation, Sov. Math. Dokl. 32 (1985) 254.

[12] V.G. Drinfel'd, Quantum Groups, Proc. Int. Congr. Math., Berkeley (1986) 798. 
[13] V.G. Drinfel'd, On almost cocommutative Hopf algebras, Leningrad Math. J. 1 (1990) 321.

[14] L.D. Fadeev, N.Yu. Reshetikhin, L.A. Takhtajan, Algebra and Analysis 1 (1987) 178.

[15] M.D. Gould, J. Links, A.J. Bracken, Matrix elements and Wigner coefficients for $U_{q}\left(g l_{n}\right)$, J. Math. Phys. 33 (1992) 1008.

[16] M. Jimbo, A q-Difference Analogue of $U(g)$ and the Yang-Baxter Equation, Lett. Math. Phys. 10 (1985) 63.

[17] A. Joseph, Quantum Groups and Their Primitive Ideals, Modern Surveys in Mathematics v.29, Springer Verlag (1994).

[18] B. Jurco, Differential Calculus on Quantized Simple Lie Groups, Lett. Math. Phys. 22 (1991) 177.

[19] S.M. Khoroshkin, V.N. Tolstoy, Universal R-matrix for quantized (super) algebras, Comm. Math. Phys. 141 (1991) 599.

[20] A.N. Kirillov, N. Reshetikhin, q-Weyl Group and a Multiplicative Formula for Universal R-Matrices, Commun. Math. Phys. 134 (1990) 421.

[21] G. Lusztig, Quantum Deformations of Certain Simple Modules over Enveloping Algebras, Adv. Math. 70 (1988) 237.

[22] G. Lusztig Introduction to Quantum Groups, Progress in Mathematics 110, Birkhäuser (1993), Boston.

[23] S. Majid, Quantum and Braided Lie Algebras, J. Geom. Phys. 13 (1994) 307, hep-th/9303148. (1993).

[24] M. Rosso, Finite Dimensional Representations of the Quantum Analog of the Enveloping Algebra of a Complex Simple Lie Algebra, Commun. Math. Phys. 117 (1988) 581.

[25] M. Rosso, Analogues de la forme de Killing et du theoreme d'Harish-Chandra pour les groupes quantiques, Ann. scient. Ec. Norm. Sup. 23 (1990) 445.

[26] H. Samelson, Notes on Lie Algebras, Van Nostrand (1969).

[27] P. Schupp, P. Watts, B. Zumino, Bicovariant Quantum Algebras and Quantum Lie Algebras, Commun. Math. Phys. 157 (1993) 305.

[28] P. Schupp, Quantum Groups, Non-Commutative Differential Geometry and Applications, hep-th/9312075 (1993).

[29] P. Schupp, Cartan Calculus: Differential Geometry for Quantum Groups, hepth/9408170 (1994). 
[30] K. Schmüdgen, A. Schüler, Classification of Bicovariant Differential Calculi on Quantum Groups of Type A, B, C and D, Comm. Math. Phys. 107 (1995) 635.

[31] A. Sudbery, The quantum orthogonal mystery, in Quantum Groups: Formalism and Applications, ed. J. Lukierski, Z. Popowicz and J. Sobczyk (Polish Scientific Publishers PWN, 1995), p. 303.

[32] A. Sudbery, V. Lyubashenko, Quantum Lie Algebras of Type $A_{n}$, q-alg/9510004.

[33] M.E. Sweedler, Hopf algebras, Benjamin, New York (1996).

[34] S. Wolfram, Mathematica, Addison-Wesley Publishing Co. (2nd ed.) (1991).

[35] S.L. Woronowicz, Differential Calculus on Compact Matrix Pseudogroups (Quantum Groups), Comm. Math. Phys. 122 (1989) 125. 